\title{
Antonio Serrano Peral y la construcción de la iglesia de Nuestra Señora de Gracia en Alicante, España
}

\author{
Antonio Serrano Peral and the construction of the church of Nuestra Señora de \\ Gracia in Alicante, Spain
}

Recibido: diciembre 2019

Aceptado: septiembre 2020

\section{Resumen}

El artículo se plantea indagar en las posibles fuentes de inspiración de Serrano Peral y en los procesos constructivos de la iglesia de Nuestra Señora de Gracia deAlicante, proyectada en 1945, a partir de una triple línea metodológica que trata datos biográficos, aspectos técnicos y condiciones contextuales de la posguerra española. El estudio se ha basado principalmente en la documentación existente en el Archivo General de la Administración del Estado y en el archivo-biblioteca personal del propio autor.

\section{Palabras Clave:}

Arquitectura religiosa; Alicante; Franquismo

\author{
José Antonio Martínez Prades ${ }^{1}$
}

The article tries to investigate in the possible sources of inspiration of Serrano Peral and in the constructive processes of the church of Nuestra Señora de Gracia in Alicante, projected in 1945, from a triple methodological line that deals with biographical data, technical aspects and contextual conditions of the Spanish post-war period. The study is based mainly on the documentation existing in the General Archive of the State Administration and in the personal library-archive of the author himself.

\section{Keywords:}

Religious architecture; Alicante; Francoism

\footnotetext{
${ }^{1}$ Nacionalidad: español; adscripción: Coordinador del Área de Historia del Arte del Departamento de Humanidades Contemporáneas de la Universidad de Alicante (España); Doctor en Historia del Arte; e-mail: jantonio.martinez@ua.es
} 


\section{INTRODUCCIÓN}

La iglesia de Nuestra Señora de Gracia de Alicante constituye una de las obras de mayor envergadura de la posguerra en la ciudad y su arquitecto, la figura más importante y prolífica del panorama arquitectónico del momento. Antonio Serrano Peral (Elche, 1907-t.1935-1968) ocupó el cargo de arquitecto diocesano del Obispado de Orihuela desde 1939 y fue nombrado en 1941 vocal de la Junta Diocesana de Reconstrucción de Templos Parroquiales, desarrollando decenas de proyectos de muy diversa índole, de arquitectura civil, religiosa y, en especial, reconstrucciones. Personalidad con destacados contactos en Madrid, formó parte de la Asamblea Nacional de Arquitectos en 1940, ostentando plaza de académico correspondiente de la Real Academia de Bellas Artes de San Fernando (RABASF) desde $1946^{2}$. No obstante, y pese a ello, la mayor parte de sus proyectos no han sido aún analizados en profundidad y la bibliografía en la actualidad todavía es escasa, compuesta principalmente por estudios catalográficos, apuntes dispersos o referencias en obras generalistas (Jaén i Urbán, 1978; Candela, 1987; Candela, 1989; Candela,1990; Jaén i Urbán, 1999; Candela, 2012; Jaén i Urbán, 2012; Jaén i Urbán, 2014; Martínez Prades, 2016) ${ }^{3}$

\subsection{Objetivos}

Los objetivos se han dirigido a desentrañar el caso de uno de los edificios tradicionales de la Junta Nacional de Reconstrucción de Templos Parroquiales y a poner de manifiesto a su vez las aportaciones personalistas de su autor, muy en especial, su original forma de aunar diferentes influencias.

\subsection{Elección del templo}

La inclinación hacia este edificio viene motivada porque constituye su primera gran obra religiosa de importancia, situada en una nueva zona de urbanización de la capital alicantina de los años 40 y porque en ella se combina una arquitectura muy tradicional, inspirada en el espíritu herrerianoescurialense, tan presente en el primer franquismo, con la adaptación a los medios técnicos disponibles, incluso con el uso del hormigón armado.

\subsection{Fuentes y metodología}

Como fuentes, ha sido analizada la documentación sobre la iglesia existente en el Archivo General de la Administración (Junta Nacional del Reconstrucción de Templos Parroquiales -JNRTP-, Dirección General de Regiones Devastadas), la biblioteca personal de Antonio Serrano Peral en Elche, la bibliografía específica y la comparación gráfica. La metodología se basa en tres planos: el personal del propio autor, el técnico-arquitectónico y el contextual de la posguerra.

\section{EL PROYECTO DE SERRANO PERAL}

El caso que nos ocupa se trata de un proyecto de reedificación de la Junta Nacional de Reconstrucción de Templos Parroquiales, firmado en 1945 (Serrano, Proyecto, Memoria, 1945: 1-2). La iglesia preexistente, bajo la misma advocación, formaba parte del Monasterio de Franciscanos levantado en la zona denominada "la Montañeta" en Alicante y, según reza la Memoria adjunta al proyecto, fue fundado en los últimos años del reinado de Fernando el Católico. El convento quedó desamortizado en 1836, aunque la iglesia permaneció abierta al culto. Fue incendiada en 1936, quedando en estado de ruina $y$, si bien se considera un proyecto de reconstrucción, en realidad supone una construcción ex novo, en la que únicamente se conserva de su antecesora el nombre, pues el templo proyectado se alzó a muy poca distancia del anterior, pero en una nueva ubicación. Para ello, se aprovechó el desmonte de la Montañeta y la explanada que dejó tal operación (Figura 1), en la que se emplazaron los nuevos edificios oficiales del régimen y, entre ellos, la propia iglesia de Nuestra Señora de Gracia.

\footnotetext{
2 Titulado por la Escuela de Arquitectura de Madrid en 1935 (en 1928, como aparejador), publicó, en 1945, algunos de sus más insignes proyectos de restauración en la Revista Nacional de Arquitectura sobre la iglesia de Santa María de Elche y la iglesia parroquial de Elda, ambas en la provincia de Alicante. Datos biográficos procedentes del Currículum Vitae proporcionado por su hijo, D. Antonio Serrano Bru (en adelante, CVSP), 8 folios por una cara sin numerar.

3 Consúltese asimismo el Proyecto de Reconstrucción del autor sobre Nuestra Señora de Gracia de Alicante. Planos, febrero de 1945; proyecto, abril de 1945. Dirección General de Regiones Devastadas, Junta Nacional de Reconstrucción de Templos Parroquiales. Archivo General de la Administración del Estado (AGA), Sección 4, Fondo 081.001, Caja 3398.
} 
Figura 1. Desmonte de la Montañeta

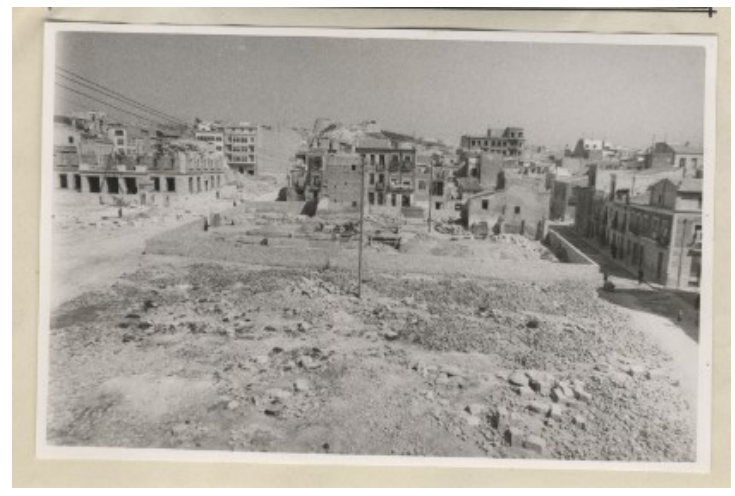

Fuente: Obras preliminares de Nuestra Señora de Gracia. Alicante. [España] Ministerio de Educación, AGA_TOP-76-04-CA-03398-015

El presente proyecto sigue la línea historicista aplicada por Serrano Peral en gran parte de su producción; los materiales estructurales son modernos, con el uso del hormigón y del ladrillo hueco, en cuya técnica el arquitecto era experto, pero en las formas y en los materiales de revestimiento, se guía por lo tradicional. Según consta en la Memoria, se proyectó trabajar con teja, mampostería, piedra labrada, pavimentación de mármol y vidrieras (Serrano, Proyecto, Memoria, 1945: 4)

En cuanto a las trazas del edificio, toma como modelo una construcción local, la Concatedral de San Nicolás, antes Colegiata, edificio de estilo herreriano. Al comparar la planta de Serrano Peral con la de San Nicolás (Figuras 2 y 3), puede constatarse la similar disposición del crucero con cúpula y las tres naves con tres tramos; tan sólo difieren en la cabecera, ajustada en esta última al primigenio diseño gótico, después abandonado. La cúpula hemisférica, paradigmática de 10 herreriano, representa claramente un elemento primordial en el nuevo proyecto, tal y como recoge el propio arquitecto en la mencionada Memoria (Serrano, Memoria, 1945: 4) ${ }^{4}$. Por otro lado, los referentes herrerianos de San Nicolás se encuentran respaldados por datos históricos, ya que sabemos que su arquitecto fue Agustín Bernardino, discípulo del mismo Juan de Herrera (Martínez Morellá, 1960: 37-38).
Figura 2. Planta de Nuestra Señora de Gracia, Alicante

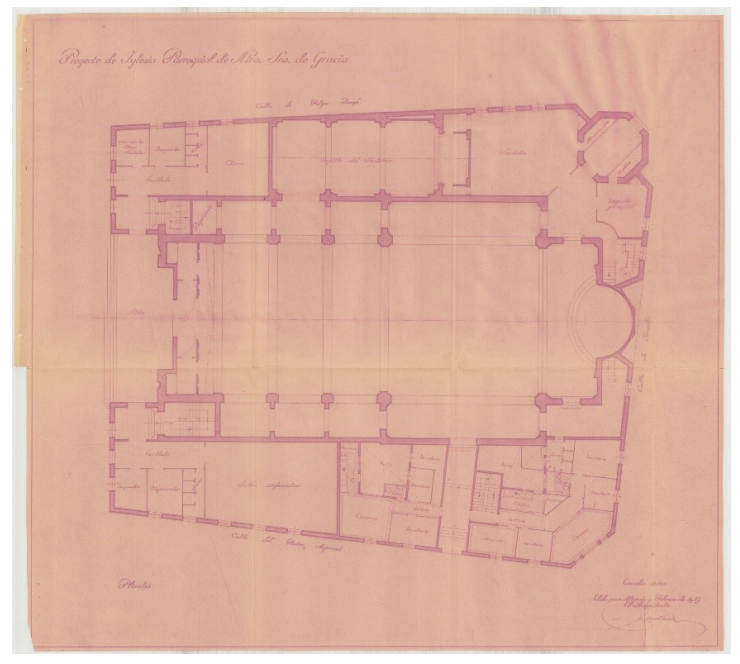

Fuente: [España] Ministerio de Educación, AGA_TOP-76-04-CA-03398-006

Figura 3. Planta de la Colegiata de San Nicolás. Alicante

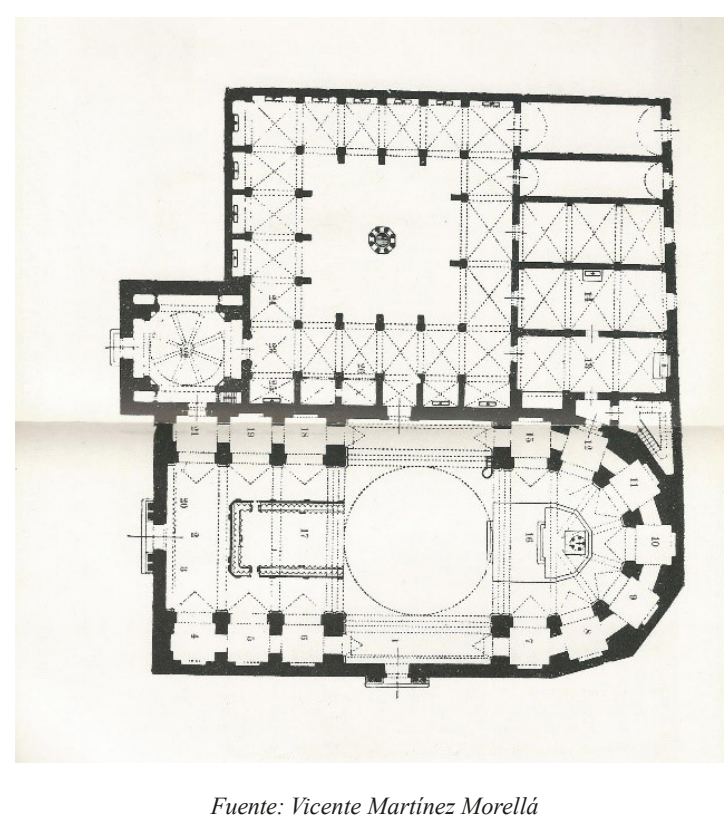

\section{LAS FUENTES DE INSPIRACIÓN}

Como se ha indicado, la obra de Serrano Peral fue realmente extensa, especialmente en el ámbito de la arquitectura religiosa, terreno en el que, como restauraciones, reconstrucciones o nuevos proyectos, llevó a cabo el diseño y construcción,

\footnotetext{
4 "Presidida por una gran cúpula (...), inspirada en la regia arquitectura de la herreriana Colegiata de San Nicolás"
} 
entre 1939 y 1962, de 28 templos 5 . Tal magnitud constructora y esfuerzo de creación contó sin duda con un abanico de fuentes inspiradoras e influencias, trabajo de investigación que, en su mayor parte, está por realizar para comprender en toda su magnitud la esencia de la arquitectura del autor ilicitano. No nos explayaremos en este punto por razones obvias, pero la amistad que le unía a importes figuras y arquitectos en Madrid y en otros lugares de España y su presencia en diferentes eventos nacionales le proporcionó una gran amplitud de miras para la elaboración de sus proyectos, tradicionales en sus inicios, pero evolucionados hacia una arquitectura más actual y personalista a partir de los años 50 .

Por otro lado, esta inquietud por la creación religiosa, hizo que el autor reuniera, en su biblioteca particular, obras litúrgicas y sobre construcción religiosa; y en este sentido, se mantenía informado de los últimos movimientos de la arquitectura cristiana contemporánea, incluso en el ámbito internacional, con publicaciones, no sólo españolas, sino también alemanas o italianas ${ }^{6}$. Estas obras le inspiraron, influyeron e incluso determinaron algunas de sus creaciones.

Deteniéndonos en este punto y volviendo a la iglesia de Nuestra Señora de Gracia que nos ocupa, a tenor del análisis de los citados fondos bibliográficos, cabe apuntar, a modo de hipótesis, una nueva lectura de su portada (Candela, 2012: $112^{7}$ ), elemento que, por otra parte, difiere del resto del templo. Su estructura podría pasar desapercibida, si no fuera por la existencia de una publicación, en la biblioteca de Serano Peral, que puede dar luz sobre sus procesos creadores. Se trata de una obra de Eduardo Junyent sobre construcción religiosa, editada en 1940 (Junyent, 1940) (Figura 9), donde se traen casos muy similares a la solución dada por Serrano Peral al enmarque del retablo escultórico, es decir, una estructura en falso frontón, con tejado a dos aguas, sobre arco de medio punto (Figura 4). En dicha publicación, véase el Proyecto de iglesia parroquial mediana (Figura 5) (Junyent, 1940: 151), sin duda inspirado en la iglesia parroquial de Aprilia (Junyent, 1940: 189) (Figura 6), aunque con la transformación de su pórtico original, con gran hornacina, en un sistema de arquivoltas; Serrano Peral incorpora el retablo a este mismo esquema, por otro lado, composición que asimismo guarda parecido a otro edificio incluido en la publicación, Santa Elena de Clignacourt (París, 1936) (Figura 7) (Junyent, 1940: 249).

\section{Figura 4. Nuestra Señora de Gracia, Alicante}

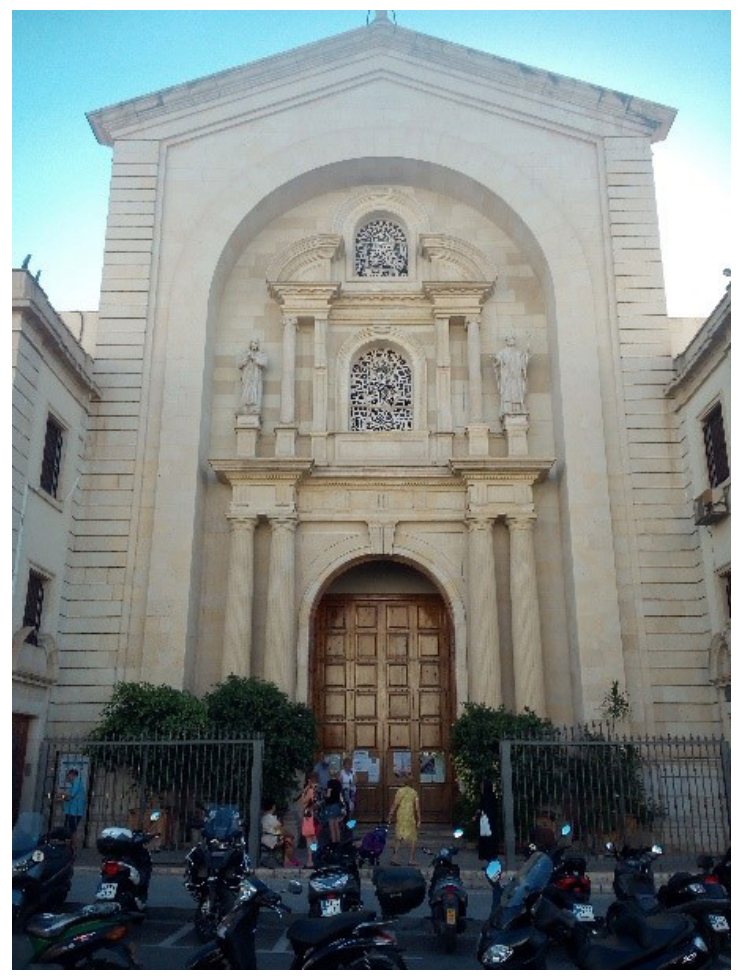

Fuente: Portada, fotografia del autor

Del mismo modo la iglesia de San Félix Cantalicio (San Felice da Cantalice, Centocelle, Roma) (Junyent, 1940: 248), aunque más alejada de estos presupuestos, conforma un gran arco central bajo un tímpano (Figura 8) y puede encuadrarse en esta misma estética. Construida entre 1934 y 1935 (Paniconi, 2014), al igual que la iglesia de Aprilia, se encuentran dentro de las construcciones que el régimen fascista italiano promovió dentro de la modernización del templo católico (Monzo, 2017 $)$.

\footnotetext{
5 Datos procedentes del CVSP y del AGA, Sección 4, Fondo 081.001.

${ }^{6}$ Biblioteca personal de Antonio Serrano Peral. Elche. Visita y fotografías facilitadas por su hijo, D. Antonio Serrano Bru (27.02.2017).

7 La portada la relaciona la autora con las portadas-retablo valencianas (San Miguel de los Reyes de Valencia).

${ }^{8}$ Véase esta obra en relación al contexto de su construcción.
} 
El retablo de Nuestra Señora de Gracia, situado en el nicho de la portada (Figura 4), plenamente herreriano con matices barrocos, está de la misma forma inspirado en la Concatedral de San Nicolás (Figura 10). Por otro lado, el susodicho enmarque de la portada lo aplicará igualmente, aunque transformado, a la Iglesia de El Salvador de Elche, proyecto de 1953 (CVSP; Candela, 2012: 164').

Figura 5. Proyecto de iglesia parroquial mediana

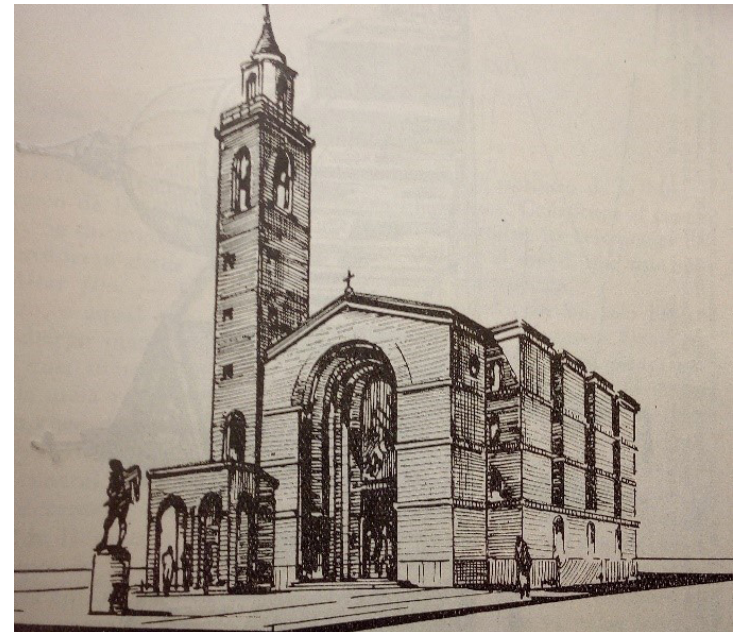

Fuente: Eduardo Junyent. Página 151

Figura 6. Iglesia parroquial de Aprilia

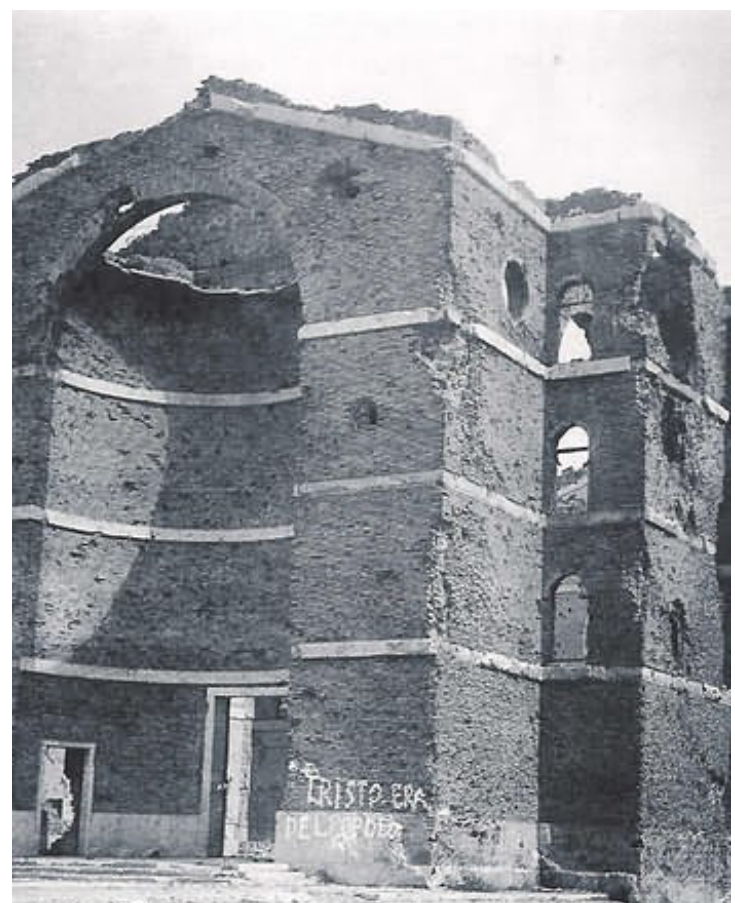

Fuente: Opera Nazionale Combattenti, 1936. Destruida en 1944. Fotografía Città di Aprilia
Figura 7. Santa Elena de Clinacourt, París, 1936

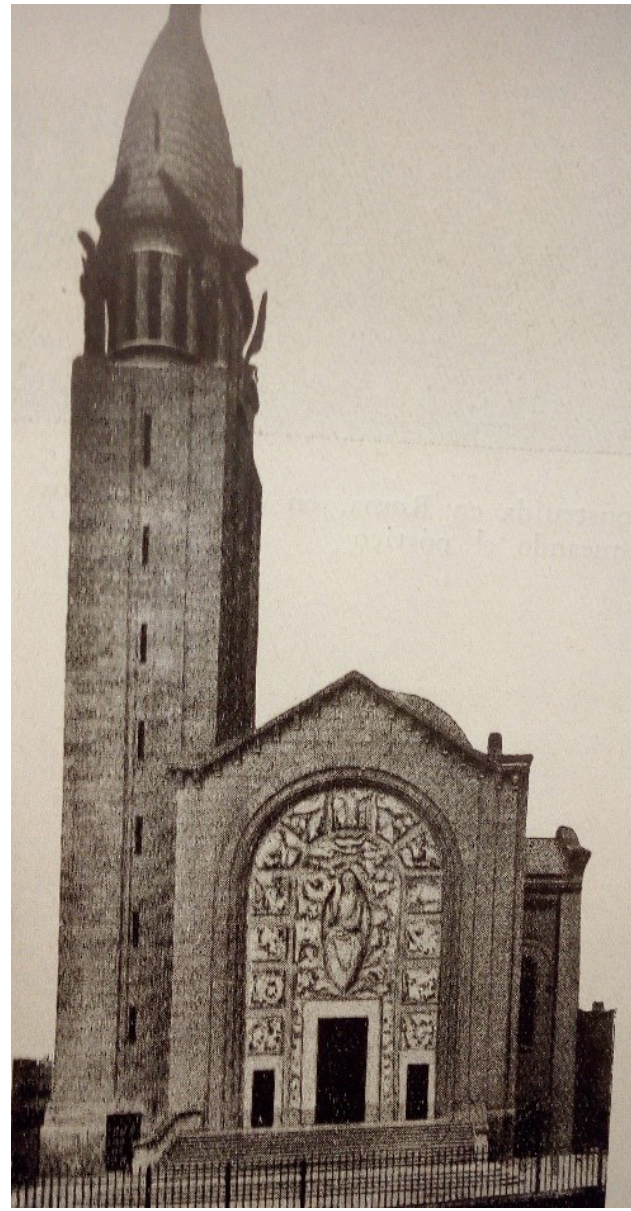

Fuente: Eduardo Junyent. Página 193

Figura 8. San Félix Cantalicio, Roma, 1934-35

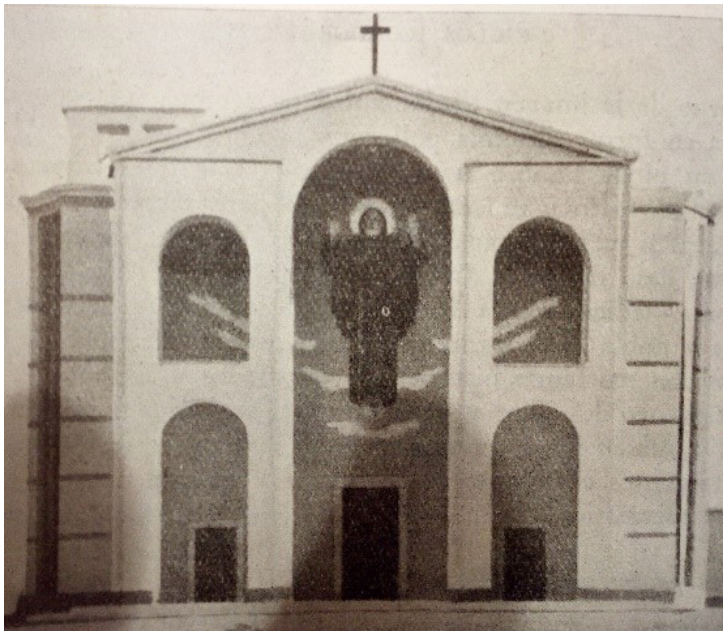

Fuente: Eduardo Junyent. Página 248

\footnotetext{
${ }^{9}$ Véase la fotografía que publica la autora.
} 


\section{ALGUNOS ASPECTOS TÉCNICOS}

La arquitectura de Serrano Peral se basó en la tradición constructiva de las obras tardobarrocas levantinas, pero acomodada a los avances contemporáneos, como el uso del hormigón armado y apoyada a su vez en un riguroso dominio técnico. Dentro de su formación, ayudaron sin duda sus contactos en Madrid con destacadas figuras del momento, como Teodoro Anasagasti, arquitecto y académico de la RABASF, con el que se relaciona en 1934 (CVSP; Candela, 2012: 18); siendo a su vez en el mismo año miembro fundador de los Seminarios de Urbanismo de la Escuela Superior de Arquitectura de Madrid, bajo de la dirección del alcoyano César Cort Botí (CVSP; Candela, 2012: 18). $Y$, en especial, con el ingeniero Eduardo Torroja, en 1939, de quien recibe estudios de especialización en construcciones de hormigón armado (CVSP). Pero quizás su capítulo más relevante estuvo constituido por sus estudios sobre bóvedas tabicadas con Luis Moya en 1940 (CVSP). Conocido es que Moya poseía unos sólidos principios tradicionales asentados en la arquitectura clásica, con base en Vitruvio y que otorgaba una gran importancia a las bóvedas de raíz romana (García-Gutiérrez, 1993: 27; Moya, $1981^{10}$ ) y que, ante la escasez de hierro y cemento, se propuso desarrollar el estudio de la técnica tradicional de las bóvedas tabicadas en su famosa obra publicada en 1927 (Moya, 1927), mostrando un gran interés por las bóvedas romanas de ladrillo y por las hemisféricas sobre pechinas (García-Gutiérrez, 1993: 62 y 86). No es casualidad que los edificios sobre los que trabaja Serrano Peral, en origen del Barroco tardío, tengan su base en el ladrillo con el uso de bóvedas sobre pechinas. Por otra parte, el arquitecto toma también contacto y gran experiencia con la realidad de este tipo de templos con la restauración de Santa María de Elche (1939) y la parroquial de Elda (1940) (Serrano, S. M. Elche, 1945: 9 y ss.; Serrano, Elda, 1945: 266 y ss. ${ }^{11}$ ), que lo colocan frente a técnicas y materiales auténticamente históricos. En el caso de Elda, detalla estos materiales de construcción, por un lado tradicionales, como por otro modernos: bóvedas de ladrillo hueco, cúpulas tabicadas con ladrillo, pero arcos de hormigón armado. (Serrano, Elda, 1945: 271).
Figura 9. Ejemplar del libro La Iglesia

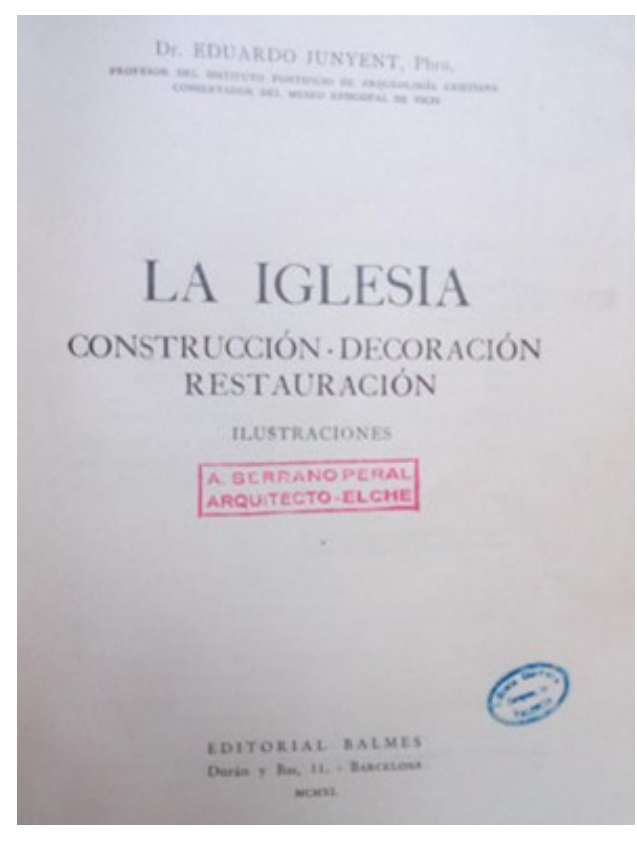

Fuente: Propiedad de Serrano Peral

Figura 10. Portada de la Concatedral de San Nicolás, Alicante

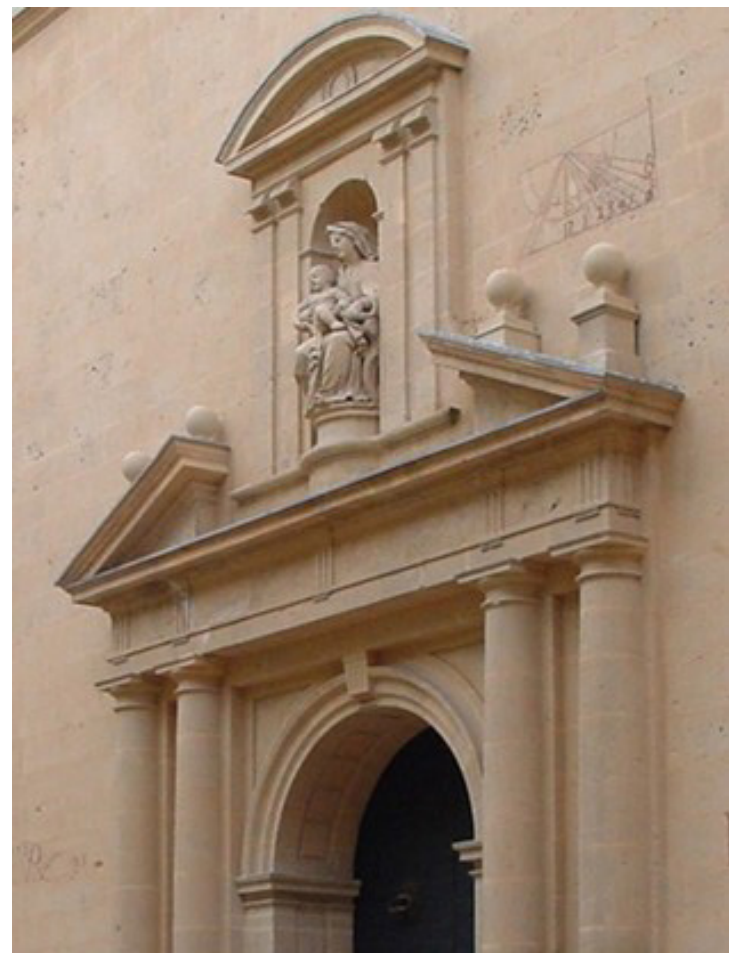

Fuente: www.iubilaeummisericordiae.va

\footnotetext{
${ }^{10}$ Aquí se recoge su discurso de entrada a la Real Academia de Bellas Artes de San Fernando en 1953, que muestra su interés por los clásicos.

11 Documentación: Archivo General de la Administración, Sección 4, Fondo 081.001, Caja 1778.
} 
Históricamente, las cúpulas tabicadas de ladrillo de varias hojas (dos o tres) fueron un tipo de construcción muy abundante en Valencia, según describe Esther Redondo siguiendo a Fornés (Álbum de proyectos de 1846, Capitulo Cúpulas dobles) ((Redondo, 2013: 124). Las mismas características presentan las del siglo XVIII, según un estudio que analiza ciertos casos de la provincia de Alicante, presentando algunas de ellas: cúpula de ladrillo de dos hojas, con la exterior ligeramente más peraltada y terminada en yeso la interior (PérezSánchez y Piedecausa, 2015: 7).

Como resulta lógico, Serrano Peral llevó a cabo la reedificación de Nuestra Señora de Gracia atendiendo a estas técnicas tradicionales, pero con la incorporación del hormigón armado. Así, en su Proyecto de Restauración, de abril de 1945, en el Estado ${ }^{\circ} 5$. Mediciones, apunta los siguientes materiales: mampostería de loseta, ladrillo macizo en pilares, hormigón armado en pilares del crucero y arcos de la nave, ladrillo de tres hojas en cúpula y bóvedas, y teja árabe (Serrano, Proyecto, Mediciones, 1945: 4-8). Incluso las proporciones de los ladrillos macizos se acercan mucho a las del ladrillo pequeño tradicional: $5 \times 12 \times 25 \mathrm{~cm}$. en Nuestra Señora de Gracia (Serrano, Proyecto, Precios $\left.{ }^{12}, 1945: 1\right)$, $3 \times 13 \times 26 \mathrm{~cm}$, medidas del tradicional (PérezSánchez y Piedecausa, 2015: 8), levantando de nuevo el desaparecido templo primigenio con respeto a las técnicas y materiales históricos.

\section{SERRANO PERAL Y LA ARQUITECTURA DE LA POSGUERRA}

Pero en la construcción del templo de Santa María, el arquitecto, no sólo tuvo en cuenta la acomodación de la técnica a la tipología del edificio y a las limitaciones económicas de la posguerra, sino algo quizás más importante, la adaptación a los principios simbólico-políticos del nuevo estado. Todo indica que su relación con la Iglesia fue estrecha, así como con altas personalidades políticas y culturales del régimen, tanto por sus contactos en Madrid, como por su actividad en la defensa del Palmeral de Elche (Gaspar, 1989: 82$\left.84^{13}\right)$. Como ya se ha mencionado anteriormente, fue nombrado arquitecto diocesano del Obispado de Orihuela en 1939 y, en 1941, vocal de la Junta Diocesana de Reconstrucción de Templos Parroquiales, moviéndose, en los años 40 , siempre dentro de unos muy tradicionales diseños, algo que, por otra parte, era propio de la JNRTP. Hemos hablado asimismo de la posesión en su biblioteca del manual de Eduardo Junyent sobre construcción religiosa editado en 1940 (Figura 9), referente, sin duda, de la estética historicista del momento (Plazaola, 1965: 72614). Por otro lado, aunque desconocemos si Serrano Peral participó en la Exposición Internacional de Arte Sacro (EIAS) de Vitoria de 1939, sí podemos asegurar que tenía conocimiento de la misma por el Catálogo presente en su biblioteca (Figura 11). La EIAS se convirtió un evento propagandístico del franquismo que intentaba presentar falsos aires de renovación en el arte sacro en contraposición al ambiente de conservadurismo religioso reinante; prueba de ello fueron las críticas levantadas entre clero y la reafirmación de este en los principios artísticos del pasado (Larrinaga, 2006: 232). También, Dos años de Arte Religioso, de 1942 (Figura 12), presente en su biblioteca, nos habla de su formación tradicionalista (Ferrando, 1942).

Tampoco podemos obviar la participación de Serrano Peral como miembro de la Asamblea Nacional de Arquitectura en 1940 y 1941, sucesoras de la I Asamblea, celebrada en 1939 bajo los auspicios de la Falange, que inició el establecimiento de las directrices que la arquitectura nacional debía seguir en su desarrollo (Gutiérrez, 1939'15: 41; Muguruza, 1939 ${ }^{16}$ : 3-13; Box, 2009; Portela, 2002). Una de las líneas surgida a partir de estos debates teóricos se basó en la tradición herreriano-escurialense, erigida en pretendido

\footnotetext{
12 Sección Precios asignados a los materiales.

13 Serrano Peral, ya desde 1933, comenzó a organizar la campaña de defensa de los palmerales ilicitanos (CVSP), hecho que le puso en contacto con numerosas personalidades que lo visitaron para conceder su apoyo a tan magno proyecto y de cuyas visitas restan diversos testimonios gráficos. El propio Franco, en 1943, firmó un decreto de declaración como Jardín Artístico.

14 Según Plazaola, Junyent “estudia los elementos integrantes de la iglesia. Omite todo lo referente al movimiento litúrgico y se muestra muy conservador y académico (...) El primer manual sobre el tema publicado en la España de la postguerra”.

15 "Estas necesidades tienen su expresión en la doctrina del Movimiento Nacional-sindicalista, que ha de realizarse en un plan nacional, en una perfecta organización que sea fuerte (...). Toda organización responde a una idea, a un fin, y éste debe ser totalitario, dictador, nacional",

16 Asimismo, se pronunciaba Pedro Muguruza en la misma Asamblea.
} 
paradigma simbólico del nuevo estado, cuyo uno de sus más conspicuos defensores fue el ideólogo fascista Ernesto Giménez Caballero (Giménez, 1935 17; Martínez, 1917).

Así pues, imbuido del espíritu político del momento, Serrano Peral diseñó el interior de Nuestra Señora de Gracia bajo el influjo de El Escorial, modelo, por otro lado, aplicado en varios casos de reconstrucción en la provincia de Alicante (Martínez 2016; Martínez, 2018). Aquí, el gran número de templos destruidos durante la contienda civil hizo muy abundantes las actuaciones de la JNRTP, cuyos proyectos de reedificaciones, en principio, se basaban en la recuperación de los antiguos edificios derruidos, generalmente del barroco tardío, pero transformados durante su construcción, prescindiendo de su

Figura 11. Portada del catálogo de la EIAS

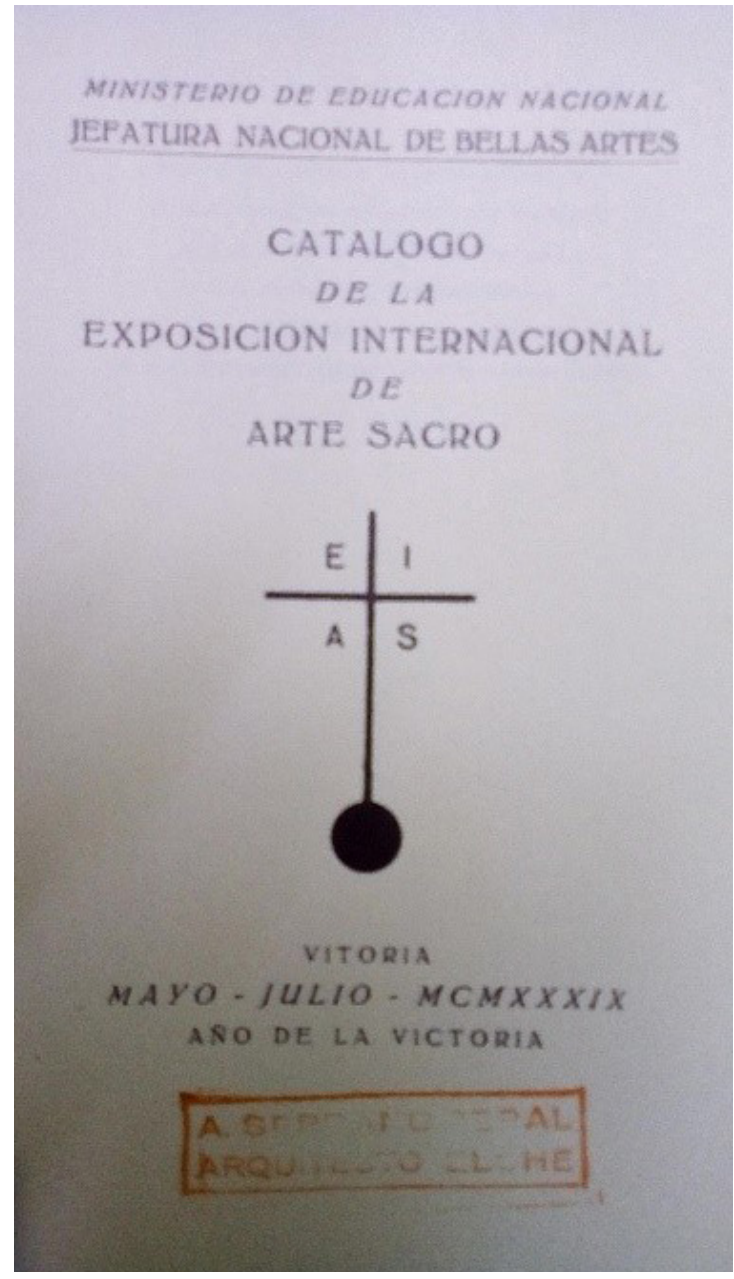

Fuente: Propiedad de Serrano Peral

\footnotetext{
${ }^{17}$ Destacamos ésta entre sus numerosas publicaciones.
}

decoración original e imprimiendo en su interior el estilo severo y el espíritu de austeridad que caracterizan a la Basílica de El Escorial, que puede constatarse en la iglesia que nos ocupa, salvo por el cambio de pilastras toscanas a jónicas (Figura 13). De este modo, queda patente el expresado deseo del arquitecto de inspirarse en la arquitectura herreriana, como se ha indicado más arriba.

Figura 12. Ejemplar del libro Dos Años de Arte Religioso

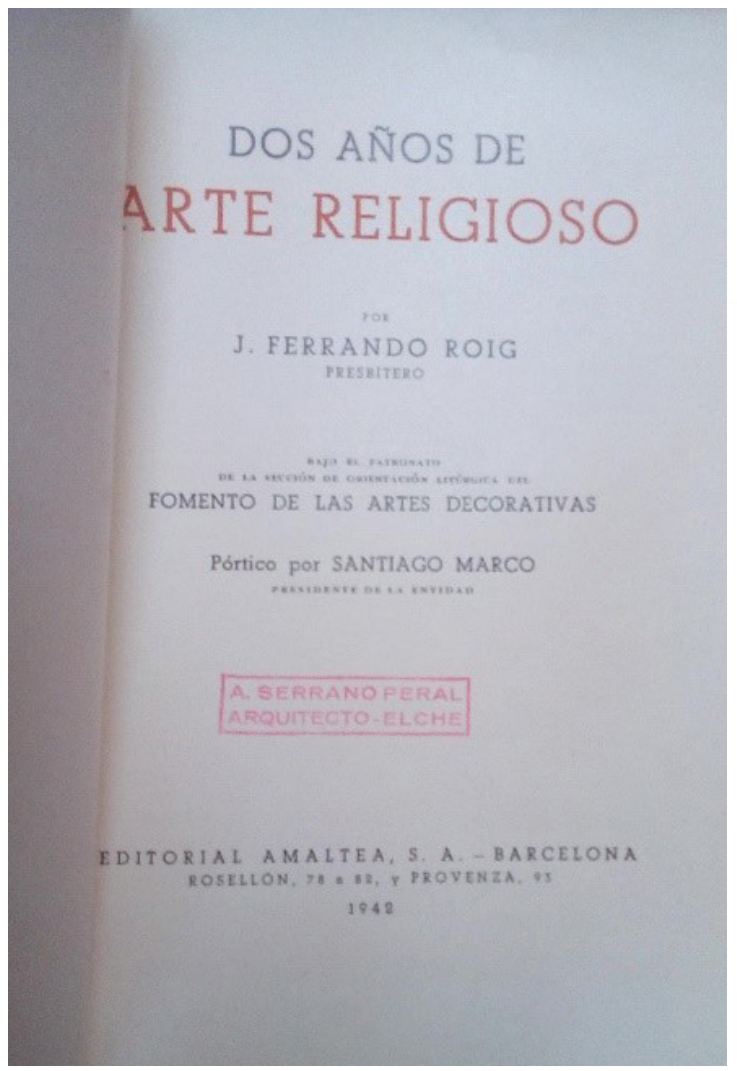

Fuente: Propiedad de Serrano Peral

\section{Figura 13. Nuestra Señora de Gracia}

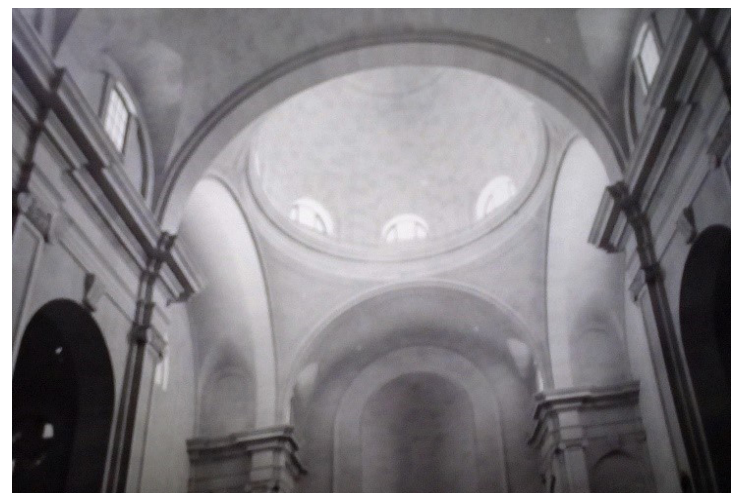

Fuente: Interior. Fotografia de A. Serrano Peral 


\section{CONCLUSIONES}

En suma, sobre la iglesia de Nuestra Señora de Gracia, puede concluirse:

1. Que dicho templo constituye su primera gran obra construida ex novo, a pesar de tratarse oficialmente de una reconstrucción, evocadora de lo herreriano, como el propio autor deja reflejado en la Memoria de su Proyecto, consiguiendo sugerir el espacio místico escurialense, que él mismo y otros arquitectos se encargarán de reproducir en la reconstrucción de otros templos.

2. La gran habilidad creadora del autor en la adaptación de unas técnicas tradicionales basadas en el ladrillo y extendidas en la arquitectura del Barroco tardío levantino, pero actualizadas con estudios contemporáneos al máximo nivel sobre sus posibilidades y problemática.

3. La combinación de estas técnicas latericias, que daban continuidad a la tradición y abarataban la construcción, con el uso del hormigón armado, que proporcionaba la solidez necesaria a un edificio de gran luz y hablaban del arquitecto como un destacado experto en su aplicación.

4. La adaptación del templo a los principios ideológicos del régimen en cuestiones de arquitectura religiosa y, en particular, a la JNRTP, siguiendo el modelo herreriano; si bien, cabe reseñar que el catálogo constructivo del arquitecto fue muy amplio y asimismo incluyó obras de arquitectura civil moderna, art déco y racionalismo.

En consecuencia, la iglesia de Nuestra Señora de Gracia constituye una amalgama de formas y soluciones constructivas inspiradas en diversos modelos e influencias, que dan como resultado un edificio con una singular portada, una apariencia general externa marcada por la tradición levantina y un interior modulado por la impronta de El Escorial. C

\section{REFERENCIAS BIBLIOGRÁFICAS}

Box Varela, Zira (2009), La fundación de un régimen: la construcción simbólica del franquismo. Tesis Doctoral. Madrid: Universidad Complutense.

Candela Garrigós, María de los Reyes (1987), Antonio Serrano Peral. Tesis de Licenciatura inédita. Sevilla: Universidad de Sevilla.

Candela Garrigós, M. R. (1989), "Un arquitecto ilicitano: Antonio Serrano Peral". Atrio, 1, 61-66.
Candela Garrigós, M. R. (1990), La obra arquitectónica de Antonio Serrano Peral (1928-1968). En Ayudas a la Investigación 1986-87. Arqueología, Arte, Toponimia. Alacant: Instituto de Cultura Juan GilAlbert. Vo. III, 233-243.

Candela Garrigós, M. R. (2012), La obra arquitectónica de Antonio Serrano Peral (1928-1968). Madrid: Editorial Dykinson.

Ferrando Roig, J. (1942.), Dos años de arte religioso. Barcelona: Editorial Amaltea.

García-Gutiérrez, Javier (Ed.) (1993), Cuaderno de apuntes de construcción de Luis Moya (1924-1925). Madrid: Instituto Juan de Herrera.

Giménez Caballero, Ernesto (1935), Arte y Estado. Madrid: Gráfica Universal.

Gutiérrez Soto, Luis (1939), Dignificación de la vida (Vivienda, Esparcimiento y Deportes). En VV.AA. 1939. I Asamblea Nacional de Arquitectos. Teatro Español de Madrid, 26, 27 y 28 de junio de 1939. Madrid: Servicios Técnicos de FET y de las JONS, Sección de Arquitectura.

Jaén i Urbán, Gaspar (1978), Guía provisional de la ciutat d'Elx. Alicante: Colegio Oficial de Arquitectos de Alicante.

Jaén i Urbán, Gaspar (dtor.) (1999), Guía de arquitectura de la provincia de Alicante. Alicante: Instituto de Cultura Juan Gil Albert.

Jaén i Urbán, Gaspar (2012), “Antonio Serrano Peral, arquitecto: viviendas, fábricas, dotaciones". Festa d'Elx, 56, 69-94.

Jaén i Urbán, Gaspar (2014), Antonio Serrano Peral (1907-1968). Arquitecto moderno heterodoxo. Recuperado de RUA http://hdl. handle.net/10045/40864

Junyent, Eduardo (1940), La iglesia, construcción, decoración, restauración. Barcelona: Balmes.

Larrinaga Cuadra, Andere (2006), "La Exposición Internacional de Arte Sacro de Vitoria de 1939: un hito artístico en la postguerra española”. Ondare. 25, 232.

Martínez Morellá, Vicente (1960), La iglesia de San Nicolás de Alicante. Alicante: Instituto de Estudios Alicantinos, 37-38.

Martínez Prades, José Antonio (2016), “La reconstrucción de la iglesia de San Juan de Elche y otras empresas neobizantinas de Serrano Peral". Archivo de Arte Valenciano, 
97, 381-392. Valencia: Real Academia de Bellas Artes de San Carlos de Valencia.

MartínezPrades, JoséAntonio(2016), Reconstrucción $y$ transformación arquitectónica en el franquismo: la Iglesia de Santa María de Alcoy (Alicante). En Sansano, Gabriel y Otros (Coor.). 2016. Congrés Internacional Història $i$ poètiques de la memòria: la violència política en la representació del franquisme. Alacant: Universitat d'Alacant, 2014, 445-459

Martínez Prades, José Antonio (2017), “La impronta de El Escorial en la arquitectura española de la posguerra: algunos casos de reconstrucciones religiosas en la provincia de Alicante (España)".Estoa. Revista de la Facultad de Arquitectura y Urbanismo de la Universidad de Cuenca, Ecuador, Vo. 6, $\mathrm{n}^{\circ} 10,51-59$.

Martínez Prades, José Antonio (2018), La reconstrucción religiosa como memoria del franquismo: casos en estudio. En Sansano, Gabriel (Ed.). 2018. Silenci, oblit i preservació de la memòria democràtica: una aportació transversal. Alacant: Universitat d'Alacant, UNE, 97-109.

Monzo, Luigi (2017), Croci e fasci: Der italienische Kirchenbau in der Zeit des Faschismus, 1919-1945. Karlsruhe: Institut Kunst-und Baugeschichte (IKB), Universität Karlsruhe. Recuperado de https://publikationen.bibliothek.kit. edu/1000071873

Moya Blanco, Luis (1927), Bóvedas tabicadas. Madrid: Dirección General de Arquitectura.

Moya Blanco, Luis (1981), "La geometría de los arquitectos griegos preeuclidianos". Separata de Academia. Primer semestre de 1981. N52. Real Academia de Bellas Artes de San Fernando de Madrid.

Muguruza, Pedro (1939), Ideas generales sobre Ordenación y Reconstrucción Nacional. En I Asamblea Nacional de Arquitectos. En VV.AA. 1939. I Asamblea Nacional de Arquitectos. Teatro Español de Madrid, 26, 27 y 28 de junio de 1939. Madrid: Servicios Técnicos de FET y de las JONS, Sección de Arquitectura.

Paniconi, Mario e Pediconi, Giulio (2014), Chiesa di San Felice da Cantalice. AechiDiap, Sapienza, Università di Roma. Recuperado de http://www.archidiap.com/ opera/chiesa-di-san-felice-da-cantalice/

Pérez-Sánchez, J. C. y Piedecausa-García, B. (2015), "Cúpulas de crucero en templos de la provincia de Alicante (s. XVII-XIX): construcción y geometría". Informes de la Construcción. Vo. 67, 1-10.

Plazaola, Juan (1965), El arte sacro actual. Estudio. Panorama. Documentos. Madrid: Biblioteca de Autores Cristianos.

Portela Sandoval, F. J. (2002), El eco del Escorial en la arquitectura española de los siglos XIX y XX. En El Monasterio del Escorial y la arquitectura. En Actas del Simposium. San Lorenzo de El Escorial:

Instituto Escurialense de Investigaciones Históricas y Artísticas, 333-372.

Redondo Martínez, Esther (2013), La bóveda tabicada en España en el siglo XIX. Tesis doctoral. Madrid: Escuela Técnica Superior de Arquitectura, Universidad Politécnica de Madrid.

Serrano Peral,Antonio (1945), Proyecto de Nuestra Señora de Gracia de Alicante. Planos, febrero de 1945; proyecto, abril de 1945. Dirección General de Regiones Devastadas, Junta Nacional de Reconstrucción de Templos Parroquiales.

Serrano Peral, Antonio (1945), "Santa María de Elche". Revista Nacional de Arquitectura, 37, 9 y ss.

Serrano Peral, Antonio (1945), "Iglesia parroquial de Elda". Revista Nacional de Arquitectura, 43, 266 y ss. Introducción 